\title{
STELLAR POPULATIONS AT LARGE REDSHIFTS
}

\author{
ROBERT W. O'CONNELL \\ Astronomy Department, University of Virginia \\ Charlottesville, VA 22903-0818 USA \\ (rwo@persues.astro.virginia.edu)
}

\begin{abstract}
It is now possible to probe stellar populations at large lookback times. The Butcher-Oemler Effect in distant clusters is unambiguous evidence for unexpectedly recent evolution of disk galaxies in dense environments, probably through starbursts induced by interactions. Recent applications of the "red envelope" test at $z \lesssim 1$ indicate that some luminous galaxies formed $\sim 10-13$ Gyr ago.
\end{abstract}

\section{Introduction}

With one exception, evidence for evolution in nature is indirect and circumstantial in that it is not based on contemporaneous observations. The exception, remarkably enough, is evidence for evolution of the universe itself. Courtesy of the vast scale of the universe compared to the finite speed of light, astronomers are able to see directly what was happening billions of years ago. However, the distant universe is faint enough that we have only recently been able to exploit this opportunity. In fact, at the time of the Vatican Conference in 1957, as Adriaan Blaauw noted in his historical review, "Nothing was said about galaxy evolution because nothing sensible could be said". Today, one can sensibly conclude that galaxy evolution has been detected, which is a result of enormous importance, but the number of other sensible remarks which can be made is perhaps limited.

"Galaxy evolution" is not a very well defined term. Workers generally do not use it to mean the evolution of individual galaxies, which has been recognized an inevitable since the beginning of galaxy research (Hubble, 1936). Instead, they mean a systematic change with cosmic time in the statistical distribution of galaxies over their global characteristics. In prin- 
ciple, evolution can be demonstrated by a change in any one property of the galaxy sample. However, much more information is required to interpret the character of that evolution, particularly if it involves stellar populations.

This statistical definition requires that we compare local $(z \lesssim 0.05$, say) samples of galaxies to distant samples, both chosen to reflect representative conditions. If we are not careful in matching the two samples, we.can readily misinterpret the evidence for evolution. Further, the physical nature of evolution can only be understood by comparing the two epochs in detail. Thus, a comprehensive assessment of our local environment is a key ingredient in evaluating the evolution of distant galaxies. This assessment is currently rather limited, and I will comment briefly on that below.

Rapidly improved technology has allowed the study of galaxies at large lookback times to blossom. Nonetheless, the distance moduli involved $(\sim 45$ at $z=1$ ) challenge even the best instrumentation. Consequently the dimensionality of stellar population analysis of distant objects has been limited mainly to the star formation history (SFH), with a few recent studies adding morphological information. Knowledge of abundances (other than interstellar gas), kinematics, or IMF's is rudimentary.

The seminal result in the field was the discovery by Butcher and Oemler (1978) that galaxy clusters at modest redshifts of $z \sim 0.4$ contained more blue objects than local clusters. Doubts about field contamination and interpretation of the colors were resolved by spectroscopy (e.g. Dressler and Gunn, 1982) and larger samples, and the "Butcher-Oemler Effect" is now regarded as unambiguous evidence for the evolution of galaxies in rich cluster environments over the past $\sim 5$ Gyr. Simultaneously, studies of field galaxies (e.g. Tyson and Jarvis, 1979) revealed a rapid increase in number to $m \sim 24$ and a shift to bluer colors, which most workers regard as good evidence for evolution in the field.

In this review, I will concentrate on observations of rich clusters, which provide the best fiducial environment available. There are many interesting aspects of high redshift populations to which I cannot do justice, including very distant radio galaxies, gravitational lensing, and the magnituderedshift relation. .

\section{Evolution of Disk Galaxies}

\subsection{POPULATIONS IN LOCAL DISK SAMPLES}

Disk galaxies (i.e. Hubble types later than S0) constitute $60-70 \%$ of field and group samples; only in clusters does their proportion drop below $50 \%$ (Oemler, 1992). However, despite their abundance (and the fact that we live in one), we have a relatively poor understanding of their evolution, at least on the global scale relevant for comparison with distant galaxies. 
There are both fundamental and technical reasons for this. First, these are multicomponent systems, and their integrated properties are influenced by two very different populations: the disk and the bulge. Even within disks, there can be strong gradients in SFH and metallicities.

On the technical side, we have probably been misled by the ease with which we can deduce the current massive star formation rate (e.g. from emission lines). This applies only to ionizing populations, which have ages $\lesssim 5 \mathrm{Myr}$, or $0.05 \%$ of a galaxy's lifetime. The other $99.95 \%$ is a tougher proposition, and the most readily available input data-broad-band colorsare inadequate to the task. Broad-band colors can constrain the gross SFH (e.g. Gallagher et al., 1984), but it is now obvious that they contain far too little information to be useful in resolving the details of interest (e.g. smooth star formation vs. bursts vs. quenching) in the context of the early universe. Although the colors of most disks are consistent with smooth, exponentially declining SFH's in very old systems (e.g. Larson and Tinsley, 1978), they are also consistent with a variety of radically different histories (Tinsley, 1980; Schweizer and Seitzer, 1992; Fritze-v. Alvensleben and Gerhard, 1993).

Since broad-band measures seriously under-constrain stellar populations, analyses using them must provide for automatic exploration of the full alternative solution space. One method was described by Wu et al. (1980), but such algorithms have been little used. A good example of the controversies which can arise in their absence is the debate over the age of distant radio galaxies such as $0902+32$ (Lilly, 1988; Chambers and Charlot, 1990).

There are few published studies on the general ability of integrated light measures to infer the SFH of multi-component populations. One useful benchmark is the work of Pickles and van der Kruit (1990), who found that $\mathrm{S} / \mathrm{N}>20$ in $13.5 \AA$ bins between 3600 and $10000 \AA$ was needed to distinguish three population components, each specified by their age and metallicity. That is, a problem involving 6 unknowns required over 70 times as many data points. When strong redundancies are eliminated, the data requirements can probably be significantly reduced. However, the fact that stellar energy distributions are not mathematically independent (cf. Silva, 1991), which is the root cause of the problem here, implies that high precision, multi-band spectrometry is essential for analysis of composite populations. Only recently has such data become available with large apertures on a large sample of disk galaxies (Kennicutt, 1992), and their implications for the SFH have yet to be systematically explored. 


\subsection{DISTANT FIELD AND CLUSTER DISK SYSTEMS}

The smooth, exponential SFH's which were favored for spirals ten years ago predict little change in spectral properties with redshift until one reaches almost the formation epoch (e.g. Tinsley, 1980). Field counts seem consistent with this sort of "mild" evolution (Bruzual and Kron, 1980; Tyson, 1988), with no evidence for a unique formation epoch at $z \lesssim 5$. But spectra for $m \lesssim 23$ (e.g. Lilly et al., 1991; Broadhurst et al., 1992) yield a surprisingly small $\bar{z} \sim 0.4$ and few high redshift $(z \gtrsim 0.75)$ objects. This implies a major change in field properties by $z \sim 0.5$ (a $2-5 \times$ increase in the number of blue objects with $\mathrm{L}<\mathrm{L}^{*}$ ); the spectra also suggest an enhancement of starburst activity. The best explanation is non-conservation of galaxy numbers, probably indicating widespread merging since $z \sim 1$ of smaller objects into larger ones with accompanying starbursts. This is controversial, however, and the "conservative" school argues that the observations can be explained without evolution of any kind, within the uncertainties of local samples and population modeling (Koo and Kron, 1992, Koo et al., 1993). My impression is that the no-evolution models do not fit the field data well despite rather extreme input assumptions. However, this is the subject of other reviews in this volume (Ellis and Gardner), so I will not discuss it further.

In the clusters, the changes found at modest redshifts by Butcher and Oemler (1978), namely an increase in the fraction of galaxies which are $\gtrsim 0.2$ mags bluer in (B-V) than typical E/S0 objects, were also unanticipated in the "mild" evolutionary models. In nearby clusters, the blue fraction is only $\sim 2 \%$, whereas at $z \sim 0.5$ it is $\sim 30 \%$. The distant clusters are actually similar to the nearby field, and we now recognize that it is an environmentally-driven loss of this blue population in nearby dense clusters which defines the Butcher-Oemler Effect (Oemler, 1992; Dressler et al., 1994).

The broad-band color range of the blue systems is comparable to that of local, normal spirals. It was therefore initially presumed that they are normal disk galaxies. Only recently has high resolution imaging $\left(\$ 0.5^{\prime \prime}\right)$ with CFHT and HST confirmed that they are indeed predominantly disks (Lavery et al., 1992; Couch et al., 1994; Dressler et al., 1994). However, spectroscopy (reviewed by Dressler and Gunn, 1990) reveals that some $80 \%$ are not normal disk galaxies. Many appear to have abnormally high star formation rates (starbursts) or to be in a quenched phase $\sim 1-3 \mathrm{Gyr}$ following a burst, so that the main sequence includes $\mathrm{A}$ stars but does not extend to more massive types.

Couch and Sharples (1987) illustrated a particularly useful diagnostic of the blue Butcher-Oemler systems. They plot the net equivalent width of $\mathrm{H} \delta$, 


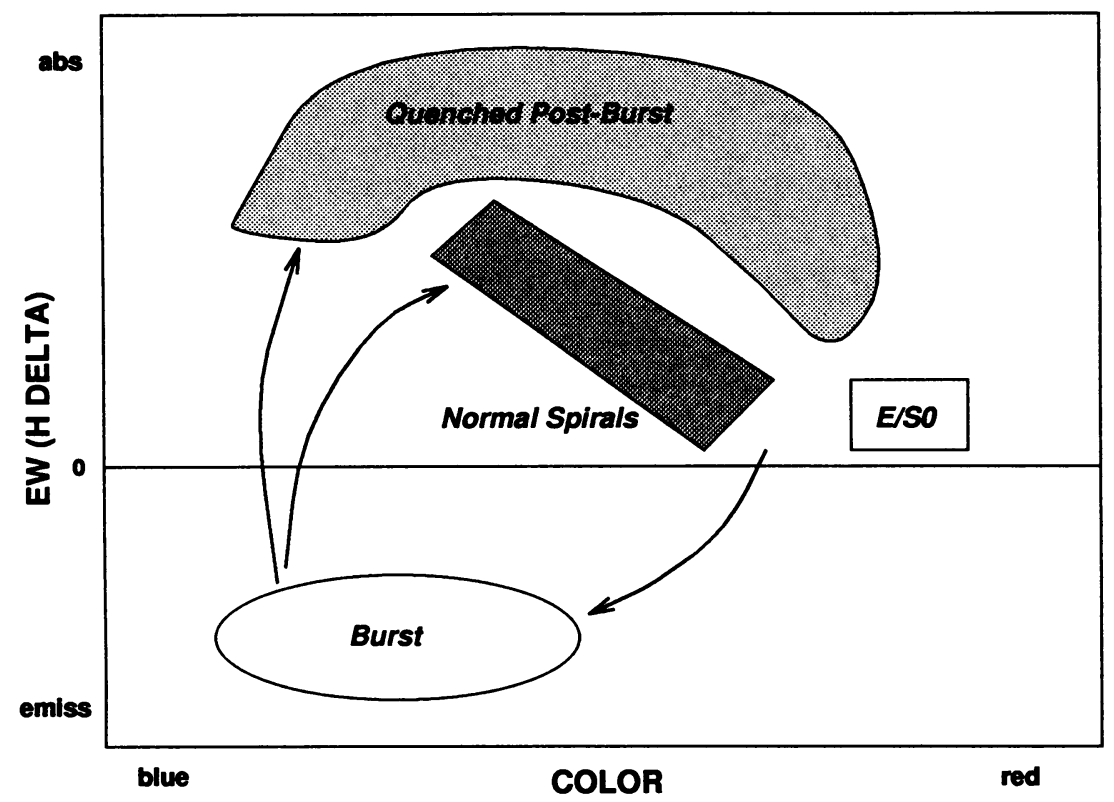

Figure 1. The location of normal galaxies and burst/post-burst systems in a Balmer line vs. color diagram. The arrows show evolutionary trajectories during and after a starburst. "Normal spirals" have smoothly declining star formation rates. In "quenched" systems normal star formation is suppressed after the burst.

measured with $\lesssim 10 \AA$ resolution, against color (see Fig. 1). In this diagram, the burst and quenched systems are well separated from normal spirals and quiescent E/S0's. Although $\mathrm{H} \delta$ is used here, a number of spectral features in the $3500-4500 \AA$ region would yield similar results. Broad-band colors, however, would not suffice.

The unusual histories implied by the spectroscopy combined with the recent imaging, which shows that many blue galaxies appear disturbed or are in interacting pairs, strongly implicate interactions or mergers in the Butcher-Oemler Effect. Not all peculiar objects have close companions (Dressler et al., 1994), but this does not necessarily exclude interactions, as shown by the local example of M81/M82. The spiral galaxy M81 has almost certainly induced the starburst now observed in M82's core; yet neither appears seriously disturbed at low resolution in the optical (except at $\mathrm{H} \alpha$ ), and they are separated by $39 \mathrm{kpc}$ (or $7^{\prime \prime}$ at $z \sim 0.3$ ). The outer parts of M82 have a quenched spectrum. This is a good example of a lingering starburst, as discussed by Schweizer in this volume.

Whether interactions or mechanisms such as IGM ram-pressure (Oem- 
ler, 1992; Dresssler and Gunn, 1990) drive the Butcher-Oemler Effect, the destruction of cold gas disks was well advanced by $z \sim 0.5$ (lookback time $\sim 5 \mathrm{Gyr}$ ) in rich cluster environments. The final products will be gas-poor, quiescent spirals and S0's (abundant in nearby clusters) and, if mergers are common, ellipticals. The radial distributions discussed by Couch and Sharples (1987) suggest that E/S0's are the most common descendents.

\section{Evolution of E/S0 Systems}

\subsection{LOCAL ELLIPTICALS}

Any attempt to understand the histories of elliptical galaxies is bedeviled by a dichotomy of properties. On one hand, there is impressive evidence for homogeneity among ellipticals, for instance the good correlations between luminosity and color (Bower et al., 1992) and between velocity dispersion and the $\mathrm{Mg}_{2}$ line index (Bender et al., 1993). The traditional emphasis has been on such regularities, which appear to argue for a single history for all E's, as described by Renzini and Franx at this conference. On the other hand, there is equally strong evidence, beginning with indications that low-luminosity ellipticals in the Local Group contained intermediate age populations (Aaronson and Mould, 1980; O'Connell, 1980), for heterogeneity and consequently for diverse histories. Insofar as integrated light is concerned, interpretations are once again strongly influenced by whether one uses broad-band colors or higher resolution spectra (e.g. Rose, 1994). I will not give details here, since they have been covered in recent reviews (Freedman, 1994; O'Connell, 1994) and were the subject of papers at this conference by Bender, Faber, Freedman, and Schweizer.

The local evidence for diversity demonstrates that star formation in $\mathrm{E} / \mathrm{S} 0$ galaxies can be, though is not always, prolonged (to $t \lesssim 5 \mathrm{Gyr}$ ago) and episodic and is often driven by environmental effects. Indeed, that would be entirely consistent with the high redshift data, as described above. The dichotomy of properties arises at least partly from an environmental effect: in denser regions, E/S0 galaxies appear to have evolved faster (Lucey, this conference). This does not entirely explain the regularities, as Renzini and Franx have properly emphasized. Nonetheless, the evidence is strong that $\mathrm{E} / \mathrm{S} 0$ galaxies are not uniformly old systems which evolve in isolation.

\subsection{EVOLUTION OF THE RED ENVELOPE}

The "red envelope" is one of the phenomena suggesting the homogeneity of $\mathrm{E} / \mathrm{S} 0$ galaxies. It is an empirical upper bound on the colors of galaxies (Sandage and Visvanathan, 1978; O'Connell, 1988). At low redshift the envelope lies at $(\mathrm{B}-\mathrm{V}) \sim 1.0$, it is well defined in rich clusters, and it is 
occupied preferentially by luminous E/S0 galaxies. This immediately suggests a test of evolution and galaxy ages: how does the envelope change with redshift?

Properly speaking, this does not test the evolution of elliptical galaxies since morphologies are mostly unknown at $z \gtrsim 0.2$. The red envelope is occupied by the least active subset of galaxies irrespective of morphology. In the absence of abnormalities (e.g. AGN's, dust, line emission, unusual IMF's, etc.), red envelope systems have experienced the smallest amount of star formation during the preceding $\sim 3 \mathrm{Gyr}$. It is incorrect to assume, as is not uncommon, that all red envelope systems are $\mathrm{E} / \mathrm{S} 0$ galaxies or that all $\mathrm{E} / \mathrm{S} 0$ galaxies fall near the envelope. It is also misleading to use "E/S0" to designate spectral or color classifications of distant galaxies whose morphologies are not known.

The red envelope test on quiescent systems is important because it is very difficult to place limits on the early SFH of galaxies with recent star forming activity, such as those discussed in $\S 2$. A schematic version of the test is shown in Fig. 2. One uses a color or gross spectral feature such as the $4000 \AA$ Break, preferentially measured in the rest frame of the distant galaxies to avoid "k-corrections". The test is empirical and differential, so broad-band measures are suitable. Current evolutionary synthesis models (e.g. Bruzual and Charlot, 1993; Worthey, 1994; Bressan et al., 1994) agree on the rate of change of such colors even if they don't agree on absolute values (Worthey, 1994). For the case shown, the models predict $\partial(U-$ $J) / \partial \log t \sim 1.4 \mathrm{mag} /$ dex. Despite its simplicity, there are many obstacles to applying the test (cf. Aragón-Salamanca et al., 1993). One complication with likely astrophysical content is the occurrence of "red interlopers"objects with colors too red for a population younger than the age of the universe and therefore probably reflecting an abnormality such as dust or an AGN.

Several groups have now applied the red envelope test to distant clusters and red field galaxies with $z \lesssim 0.9$. Bruzual and Charlot (1993) have summarized $4000 \AA$ Break data from Hamilton (1985) and Spinrad (1986). There is a significant decrease with redshift in the mean and maximum strength of the Break (implying younger populations), a result confirmed by the independent data set of Dressler and Gunn (1990). Departure of the envelope from the no-evolution prediction occurs at $z \sim 0.5$. Using the evolutionary models to age-date the Break values, one finds that $3 / 4$ have formation redshifts above 1 , which implies a present age of $t \gtrsim 10$ Gyr for $\mathrm{H}_{0}=50$ and $\mathrm{q}_{0}=0$. The envelope itself corresponds to present ages of 13-16 Gyr.

A systematic approach has been taken by Aragón-Salamanca et al. (1993), who measured broad-band $\mathrm{V}, \mathrm{I}, \mathrm{K}$ colors in 10 distant rich clusters 


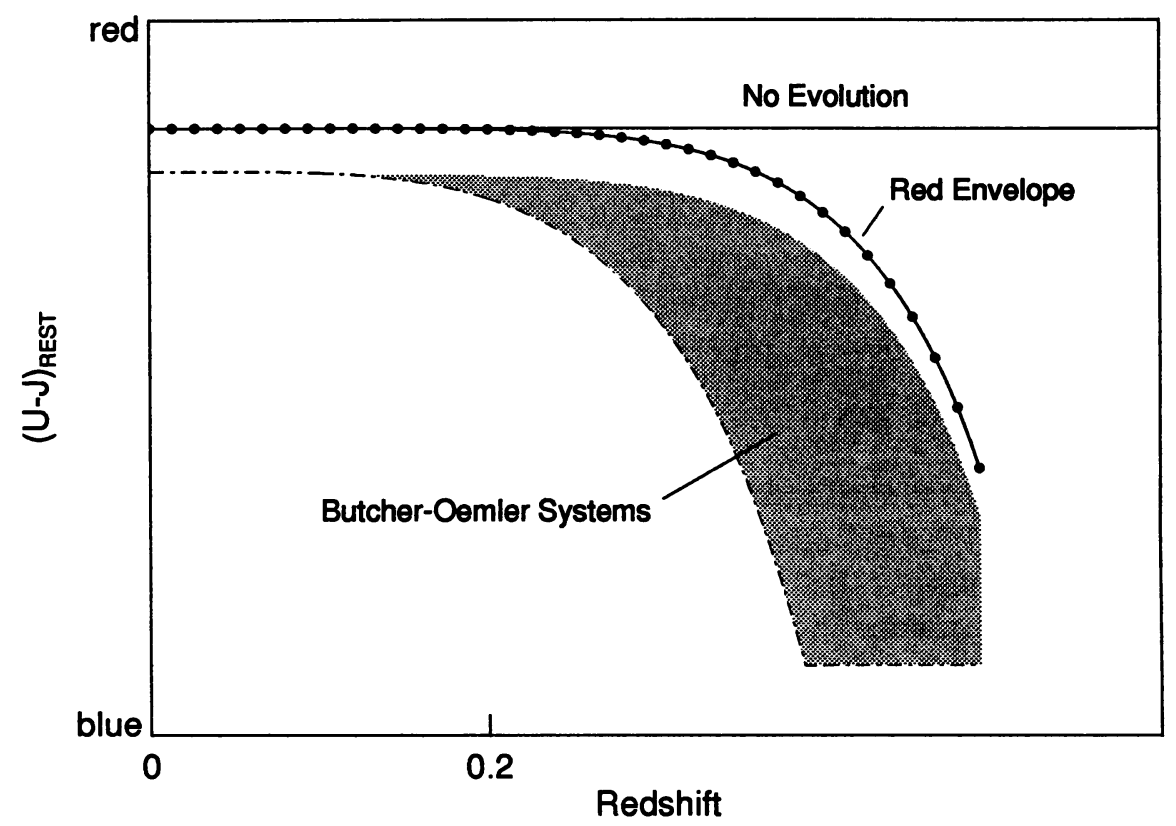

Figure 2. The red envelope test, illustrated for a long-baseline color measured in the rest frame. The dashed-dotted line is the lower boundary of colors for luminous galaxies in rich clusters. The dotted line is the upper bound, or red envelope. One measures the departure of the upper bound from the "no-evolution" line and also its slope at high redshift. The shaded area shows Butcher-Oemler systems.

up to $z=0.92$. They find a clear and systematic trend of envelope color with redshift, again departing from the no evolution expectation at $z \sim 0.5$. By $z \sim 0.9$, the envelope is almost undefined; galaxies scatter in color, and the characteristic red peak with a blueward Butcher-Oemler tail has almost vanished. The envelope trend corresponds to a formation redshift $\sim 2$, or a present age of $\sim 13 \mathrm{Gyr}$. The FWHM in the peak at $z=0.7$, after removing measurement error, corresponds to a factor of 3 in rest frame age, or a minimum present age of $\sim 10 \mathrm{Gyr}$.

Many detections of red, high $z$ objects are now being reported in various deep fields. The red interlopers in nearer clusters suggest that these systems may not always be dominated by unextincted starlight. Recent spectroscopy of high redshift radio galaxies (Hammer et al., 1993) reveals AGN-like continua in some cases and demonstrates that caution is needed in trying to age-date distant field systems from their colors. 


\section{Conclusion}

Strong evolutionary processes have transformed stellar populations in galaxies in the relatively recent past ( $\$ 5 \mathrm{Gyr})$. The evidence is unambiguous in the case of distant rich clusters, where disk galaxies are undergoing starbursts, probably induced by encounters or mergers. It is strong for field disks as well. That this was largely unanticipated from early studies of nearby galaxies demonstrates the inadequacy of our older tools for population analysis, particularly those based on broad-band colors.

No evidence for a unique galaxy formation epoch has yet emerged. Definite trends in spectral properties with redshift indicate that the least active luminous systems formed $\sim 10-13 \mathrm{Gyr}$ ago (for $\mathrm{H}_{0}=50$ and $\mathrm{q}_{0}=0$ ). Ages from such "red envelope" tests do not necessarily apply to all elliptical galaxies or to the progenitors of E/S0's in the local, low density environment. Judged from both the local and distant samples, galaxy evolution appears to be faster in denser environments.

This research has been supported in part by NASA Long Term Space Astrophysics grant NAGW-2596.

\section{References}

Aaronson, M. and Mould, J., 1980, Ap. J. 240, 804

Aragón-Salamanca, A., Ellis, R.S., Couch, W.J. and Carter, D., 1993, M. N. R. A. S. 262, 764

Bender, R., Burstein, D. and Faber, S.M., 1993, Ap. J. 411, 153

Bower, R.G., Lucey, J.R. and Ellis, R.S., 1992, M. N. R. A. S. 254, 601

Bressan, A., Chiosi, C. and Fagatto, F., 1994 Ap. J., in press

Broadhurst, T.J., Ellis, R.S. and Glazebrook, K., 1992, Nature 355, 55

Bruzual, G., and Charlot, S., 1993, Ap. J. 405, 538

Bruzual, G. and Kron, R.G., 1980, Ap. J. 241, 25

Butcher, H. and Oemler, A., 1978, Ap. J. 219, 18

Chambers, K.C. and Charlot, S., 1990, Ap. J. 384, L1

Couch, W.J. and Sharples, R.M., 1987, M. N. R. A. S. 229, 423

Couch, W.J., Ellis, R.S., Sharples, R.M. and Smail, I., 1994, Ap. J. 430, 121

Dressler, A. and Gunn, J.E., 1982, Ap. J. 263, 533

Dressler, A. and Gunn, J.E., 1990, Evolution of the Universe of Galaxies, ed. R.G. Kron, San Francisco: ASP, p. 200

Dressler, A., Oemler, A., Butcher, H.R. and Gunn, J.E., 1994, Ap. J. 430, 107

Freedman, W.L., 1994, The Local Group, CTIO/ESO, in press

Fritze-v. Alvensleben, U. and Gerhard, O.E., 1994, A. \& $A$., in press

Gallagher, J.S., Hunter, D.A. and Tutukov, A.V., 1984, Ap. J. 284, 544

Hamilton, D., 1985, Ap. J. 297, 371

Hammer, F., Le Fèvre, O. and Angonin, M.C., 1993, Nature 362, 324

Hubble, E., 1936, The Realm of the Nebulae, New Haven: Yale Univ. Press

Kennicutt, R.C., 1992, Ap. J. 388, 310

Koo, D.C., Gronwall, C. and Bruzual, G., 1993, Ap. J. 415, L21

Koo, D.C. and Kron, R.G., 1992, Ann. Rev. Astron. Astroph. 30, 613

Larson, R.B. and Tinsley, B.M., 1978, Ap. J. 219, 46 
Lavery, R.J., Pierce, M.J. and McClure, R.D., 1992, A. J. 104, 2067

Lilly, S.J., 1988, Ap. J. 333, 161

Lilly, S.J., Cowie, L.L. and Gardner, J.P., 1991, Ap. J. 369, 79

O'Connell, R.W., 1980, Ap. J. 236, 430

O'Connell, R.W., 1988, Towards Understanding Galaxies at High Redshift, eds. A. Renzini \& R.G. Kron, Dordrecht: Kluwer, p. 177

O'Connell, R.W., 1994, Nuclei of Normal Galaxies: Lessons from the Galactic Center, ed. R. Genzel, Dordrecht: Kluwer, in press

Oemler, A., 1992, Clusters and Superclusters of Galaxies, ed. A.C. Fabian, Dordrecht: Kluwer, p. 29

Pickles, A.J. and van der Kruit, P.C., 1990, A. \& A. Suppl. 84, 421

Rose, J.A., 1994, A. J. 107, 206

Sandage, A. and Visvanathan, N., 1978, Ap. J. 223, 707

Schweizer, F. and Seitzer, P., 1992, A. J. 104, 1039

Silva, D.R., 1991, PhD Thesis, Univ. of Michigan

Spinrad, H., 1986, P. A. S. P. 98, 269

Tinsley, B.M., 1980, Ap. J. 241, 41

Tyson, J.A., 1988, A. J. 96, 1

Tyson, J.A. and Jarvis, J.F., 1979, Ap. J. 230, L153

Worthey, G., 1994, Ap. J. Suppl., in press

Wu, C.-C., Faber, S.M., Gallagher, J.S., Peck, M. and Tinsley, B.M., 1980, Ap. J. 237, 290

BALCELLS: The blue galaxies with peculiar, post-starburst spectra which you mentioned-are they morphologically normal spirals, or are they morphologically peculiar objects such as those HST has unveiled? In other words, can we link the post-starburst spectra to merger remnants?

O'CONNELL: The statistics are still rough, since different groups use different classification criteria. If I combine the results for confirmed members in the three rich clusters from the Dressler et al. (1994) and Couch et al. (1994) studies, I find that $70 \%$ of the blue objects with burst or post-burst spectra are classified as interacting or merging but that only $35 \%$ of the redder objects with postburst anomalies are in these categories. Since the redder objects (called "E+A" by Dressler et al.) have presumably been disturbed less recently, this pattern is sensible. For a small sample of field galaxies, Lilly $(1993$, ApJ, 411, 501) finds that half of the blue objects are disturbed. So, interactions or mergers are probably responsible for a good deal of the activity.

FERGUSON: I think it is important to remember in these discussions of mergerinduced or tidally triggered star formation that there are many examples, especially among local dwarfs, of galaxies with star-burst behavior but no obvious neighbor to trigger the burst.

O'CONNELL: Yes, it's certainly true that rather intense star-forming activity can occur in the apparent absence of interactions. However, the examples of M81/M82 and other "lingering" starbursts of the kind Schweizer discussed, show that recent interactions aren't necessarily obvious. The HST observations of Butcher-Oemler clusters indicate that a significant fraction of the blue objects are caught during interactions. If you allow for the relatively short lifetime of strong disturbances, you find that a large fraction of the total population in a rich cluster undergoes interactions/mergers in a Hubble time. On the other hand, in the low-density field, the expected rate of interactions may be low enough to raise questions about the merger interpretation. 